J. PHYS. C (PROC. PHYS. SOC.), 1968, SER. 2, VOL. 1. PRINTED IN GREAT BRITAIN

\title{
The shapes of inhomogeneously broadened resonance lines II. Second-order effects
}

\author{
A. M. STONEHAM
}

Theoretical Physics Division, Atomic Energy Research Establishment, Harwell, Didcot, Berks.

MS. received 8th December 1967

\begin{abstract}
Resonance lines may be broadened by random strains, local electric field or similar perturbations. In simple cases the resonance line shifts linearly with the local strain or electric field; such cases have been treated in an earlier paper. In more complex cases there is no linear shift and the transition energy contains terms quadratic in the perturbation. Such cases occur frequently in spin resonance and nuclear magnetic resonance. These cases cannot be treated by the method used in the linear problem. The present paper describes an approximate method which deals with the quadratic terms. The method is a development of the powerful 'statistical method' and can be extended to still higher orders. It is compared with various other approaches to the second-order problem and illustrated by an idealized model and applied to broadening by dislocations. It is shown that dislocation-broadened lines have a width and shift linear in the dislocation density and that the line shape changes as the dislocation density is varied.
\end{abstract}

\section{Introduction}

Sharp resonance lines in solids are often inhomogeneously broadened as a result of random fluctuations of the local strain fields, local electric fields or other perturbations. In this paper we discuss the calculation of the line shape in terms of the properties of the defects which give rise to the perturbations - the statistical properties of their distribution, the perturbation fields of the individual defects and their concentration.

One general class of cases has been discussed in the literature before (see, for example, Stoneham (1966, to be referred to as I), whose notation we shall follow). In this case the transition energy of one of the centres under study shifts linearly with the local perturbation $\epsilon$ :

$$
\hbar \omega=\hbar \omega_{0}+\hbar \omega_{1} \epsilon .
$$

Here $\epsilon$ is some linear combination of the components of the local strain tensor or of the local electric field, for example. The unperturbed energy is $\hbar \omega_{0}$, and $\hbar \omega_{1}$ is a coupling coefficient. Many of the cases of interest practically follow (1). However, in certain circumstances $\hbar \omega_{1}$ is zero (usually for reasons of symmetry) or very small, so that the transition energy only changes from second-order terms:

$$
\hbar \omega=\hbar \omega_{0}+\hbar \omega_{2} \epsilon^{\prime} \epsilon^{\prime \prime} .
$$

This paper will discuss the case described by (2). Equations of this type hold for the strain broadening of the $\Delta M=2$ and double-quantum electron paramagnetic resonance lines of $\mathrm{MgO}: \mathrm{Fe}^{2+}$ (McMahon 1964), for various cases of broadening by unresolved hyperfine interaction (see Markham 1966, chap. VII, for details) and for broadening by the quadrupole interaction in nuclear magnetic resonance (Kawamura et al. 1956).

In both the linear case (1) and the second-order situation (2) we make two important physical assumptions. First we assume that the contributions of the various defects to $\epsilon$ (or $\epsilon^{\prime}$ or $\epsilon^{\prime \prime}$ ) are simply additive:

$$
\epsilon\left(Z_{1}, \ldots, Z_{N}\right)=\sum_{i=1}^{N} \epsilon\left(Z_{i}\right)
$$

Here $Z_{i}$ is an abbreviation for the position of the defect with respect to the centre studied, $\mathbf{r}_{i}$, and any internal variables of the defect, $\eta_{i}$, which are relevant. Equation (3) is the 
assumption of linear elasticity in strain broadening, and should be reasonable when $\epsilon$ is less than about $10^{-2}$. As typical strain distributions have widths two orders of magnitude less this is not a very restrictive assumption. There are similar margins for other mechanisms, such as broadening by random electric fields. The second assumption concerns the distribution of the defects. The probability of a particular configuration $\left\{Z_{1}, \ldots, Z_{N}\right\}$, in which all the positions and internal variables of the $N$ defects have definite values, is $P\left(Z_{1}, \ldots, Z_{N}\right) d z_{1} \ldots d z_{N}$. We assume that this can be factorized, so that the individual defect distributions are independent of each other:

$$
P\left(z_{1}, \ldots, z_{N}\right) d z_{1} \ldots d z_{N}=p\left(z_{1}\right) d z_{1} \ldots p\left(z_{N}\right) d z_{N} .
$$

This cannot be exact, for it ignores the fact that two defects cannot be placed on top of one another. However, (4) should be reasonable at the low concentrations usually met in practice. The statistical distribution function $p(z) \equiv p(\mathbf{r}, \eta)$ is the probability that if there is a centre contributing to the resonance line at $\mathbf{R}$ then there is a defect at $\mathbf{R}+\mathbf{r}$ in state $\eta$. Clearly $p(z)$ is closely related to the pair distribution function of the defects with respect to the centres studied.

In $\S 2$ we outline the method of I and show how the distribution of $\epsilon \equiv \Sigma_{\epsilon}\left(Z_{i}\right)$ may be calculated from equations (1), (3) and (4). It is also shown that the distribution of

$$
\Omega=\epsilon^{\prime} \epsilon^{\prime \prime} \equiv \sum_{i=1}^{N} \epsilon^{\prime}\left(Z_{i}\right) \sum_{j=1}^{N} \epsilon^{\prime \prime}\left(Z_{j}\right)
$$

cannot be calculated by this method as it stands. In $\S 3$ an approximation, loosely resembling a random phase approximation, is introduced which permits the calculation of the distribution of $\Omega$. This method is compared with other approximate methods in $\S 4$, and its application is discussed subsequently. Although the new method is an approximation it contains features which are not easily included in the other methods and which can prove valuable in practice. As the method is an extension of the well-known 'statistical method' the approach may also prove useful in other branches of spectroscopy.

\section{The linear case}

The probability that the perturbation $\epsilon$ lies between $\epsilon$ and $\epsilon+d \epsilon$ is $I_{1}(\epsilon) d \epsilon$, and may be calculated from (1), (3) and (4) with no further physical assumptions. We follow the notation and method of I.

Formally we can write

$$
I_{1}(\epsilon)=\frac{1}{v^{N}} \int \ldots \int d z_{1} p\left(z_{1}\right) \ldots d z_{N} p\left(z_{N}\right) \delta\left\{\epsilon-\epsilon\left(z_{1}, \ldots, z_{N}\right)\right\} .
$$

The delta function singles out the configurations where $\epsilon\left(z_{1}, \ldots, z_{N}\right)$ has the specific value $\epsilon$; $I_{1}(\epsilon)$ is simply the fraction of the possible configurations which have this value of $\epsilon\left(z_{1}, \ldots, z_{N}\right)$. $V$ normalizes $p(z)$ as

$$
\int d z p(z)=V
$$

Using the spectral representation of the delta function

(6) can be rewritten as

$$
\delta(y)=\frac{1}{2 \pi} \int_{-\infty}^{\infty} d x \exp (i x y)
$$

$$
I_{1}(\epsilon)=\frac{1}{2 \pi}\left(\frac{1}{V}\right)^{N} \int_{-\infty}^{\infty} d x \exp (i x \epsilon) \int d z_{1} p\left(z_{1}\right) \ldots \int d z_{N} p\left(z_{N}\right) \exp \left\{i x \epsilon\left(z_{1}, \ldots, z_{N}\right)\right\} .
$$

It is at this stage that the linear and second-order cases diverge. For the linear case the 
innermost exponential factorizes as a result of $(3)$ :

and

$$
\exp \left\{i x \in\left(z_{1}, \ldots, z_{N}\right)\right\}=\prod_{i=1}^{N} \exp \left\{+i x \in\left(z_{i}\right)\right\}
$$

$$
I_{1}(\epsilon)=\frac{1}{2 \pi} \int_{-\infty}^{\infty} d x \exp (i x \epsilon)\left[\frac{1}{V} \int d z p(z) \exp \{+i x \epsilon(z)\}\right]^{N} .
$$

No such factorization occurs in the second-order case. It is convenient to introduce new definitions here. Thus

$$
J_{1}(x) \equiv \int d z p(z)[1-\exp \{+i x \in(z)\}]
$$

and

$$
\rho \equiv N / V
$$

$\rho$ is clearly a measure of the defect concentration. By direct substitution

or, in the limit of large $N$,

$$
I_{1}(\epsilon)=\frac{1}{2 \pi} \int_{-\infty}^{\infty} d x \exp (i x \epsilon)\left\{1-\frac{\rho J_{1}(x)}{N}\right\}^{N}
$$

$$
I_{1}(\epsilon)=\frac{1}{2 \pi} \int_{-\infty}^{\infty} d x \exp (i x \epsilon) \exp \left\{-\rho J_{1}(x)\right\}
$$

Equations (8), (9) and (10) give the distribution of $\epsilon$ in terms of the individual perturbation fields $\epsilon(z)$, the statistical distribution function $p(z)$ and $\rho$, the density of the defects. These results will be needed again in the following sections.

\section{Second-order theory}

The distribution in magnitude of $\Omega$ given by (5) can be written in the same form as $(6)$. The probability that $\Omega$ lies between $\Omega$ and $\Omega+d \Omega$ is thus $I_{2}(\Omega) d \Omega$, where

$$
I_{2}(\Omega)=\frac{1}{V^{N}} \int \ldots \int d z_{1} p\left(z_{1}\right) \ldots d z_{N} p\left(z_{N}\right) \delta\left\{\Omega-\Omega\left(z_{1}, \ldots z_{N}\right)\right\} .
$$

The approach of $\S 2$ can be followed unaltered to the analogue of (7), which becomes

$$
I_{2}(\Omega)=\frac{1}{2 \pi} \frac{1}{V^{N}} \int_{-\infty}^{\infty} d x \exp (i x \Omega) \int d z_{1} p\left(z_{1}\right) \ldots \int d z_{N} p\left(z_{N}\right) \exp \left\{-i x \Omega\left(z_{1}, \ldots, z_{N}\right)\right\}
$$

However, the innermost exponential does not factorize; by (3) and (5) it is

$$
\exp \left\{-i x \Omega\left(z_{1}, \ldots, z_{N}\right)\right\}=\exp \left\{-i x \sum_{i=1}^{N} \epsilon^{\prime}\left(z_{i}\right) \sum_{j=1}^{N} \epsilon^{\prime \prime}\left(z_{j}\right)\right\}
$$

which cannot be split into a product of factors each involving the coordinates of only one of the defects.

To proceed we take advantage of the fact that the first-order problem has already been solved. The exponential in (13) is split into a product of $N$ symmetrical terms $f_{i}$ :

$$
\begin{gathered}
\exp \left\{-i x \sum_{i=1}^{N} \epsilon^{\prime}\left(z_{i}\right) \sum_{j=1}^{N} \epsilon^{\prime \prime}\left(z_{j}\right)\right\}=\prod_{i=1}^{N} f_{i} \exp \left\{-i x \epsilon^{\prime}\left(z_{i}\right) \epsilon^{\prime \prime}\left(z_{i}\right)\right\} \\
f_{i}=\exp \left[-i x \frac{1}{2}\left\{\epsilon^{\prime}\left(z_{i}\right) \sum_{\substack{j=1 \\
j \neq i}}^{N} \epsilon^{\prime \prime}\left(z_{j}\right)+\epsilon^{\prime \prime}\left(z_{i}\right) \sum_{\substack{j=1 \\
j \neq i}}^{N} \epsilon^{\prime}\left(z_{j}\right)\right\}\right] .
\end{gathered}
$$


The exponent in $f_{i}$ varies as the defects take up different configurations $\left\{z_{1}, \ldots, z_{i-1}, z_{i+1}, \ldots, z_{N}\right\}$ with $z_{i}$ fixed. We can regard the exponent of $f_{i}$ as proportional to $\phi_{i}$, a new sum of components of the internal strain:

$$
\phi_{i}=\alpha_{i} \epsilon^{\prime}+\beta_{i} \epsilon^{\prime \prime}
$$

in which $\epsilon^{\prime}$ and $\epsilon^{\prime \prime}$ are the contributions to the respective strains of the $N-1$ defects other than that at $z_{i}$, and where $\alpha_{i}$ is $\frac{1}{2} \epsilon^{\prime \prime}\left(z_{i}\right)$ and $\beta_{i}$ is $\frac{1}{2} \epsilon^{\prime}\left(z_{i}\right)$. As $N$ is large we can ignore the fact that $\epsilon^{\prime}$ and $\epsilon^{\prime \prime}$ are the contributions of $N-1$ defects, rather than $N$. The distribution of the values of $\phi_{i}$ for given $\alpha_{i}$ and $\beta_{i}$ is known from first-order theory to be $I_{1}\left(\phi_{i}\right)$. The approximation we make to factorize (13) is that we replace each factor $f_{i}$ by its average over configurations $\left\{z_{1}, \ldots, z_{i-1}, z_{i-1}, \ldots, z_{N}\right\}$ appropriately weighted. Thus $f_{i} \rightarrow F_{i}$ where

$$
\begin{aligned}
F_{i} & =\int_{-\infty}^{\infty} d \phi_{i} I_{1}\left(\phi_{i}\right) f_{i} \\
& =\int_{-\infty}^{\infty} d \phi_{i} I_{1}\left(\phi_{i}\right) \exp \left(-i x \phi_{i}\right) .
\end{aligned}
$$

Direct substitution of (10) gives

$$
\begin{aligned}
F_{i} & =\int_{-\infty}^{\infty} d \phi_{i}\left[\frac{1}{2 \pi} \int_{-\infty}^{\infty} d y \exp \left(i y \phi_{i}\right) \exp \left\{-\rho J_{i}(y)\right\}\right] \exp \left\{-i x \phi_{i}\right\} \\
& =\int_{-\infty}^{\infty} d y \exp \left\{-\rho J_{i}(y)\right\}\left[\frac{1}{2 \pi} \int_{-\infty}^{\infty} d \phi_{i} \exp \left\{i(y-x) \phi_{i}\right\}\right] .
\end{aligned}
$$

Recognizing the spectral distribution of the delta function, we have

$$
F_{i}=\exp \left\{-\rho J_{i}(x)\right\} \text {. }
$$

$J_{i}(x)$ is a function of $z_{i}$ and of $x$ written explicitly as

$$
\begin{aligned}
\tilde{J}\left(x, z_{i}\right) & =J_{i}(x) \\
& =\int d u p(u)\left(1-\exp \left[-i x_{\frac{1}{2}}\left\{\epsilon^{\prime}\left(z_{i}\right) \epsilon^{\prime \prime}(u)+\epsilon^{\prime \prime}\left(z_{i}\right) \epsilon^{\prime}(u)\right\}\right]\right) .
\end{aligned}
$$

By replacing $f_{i}$ by $F_{i}$ of equation (16) the difficult term in (13) has been factorized. It is an approximation, for we average in (15) instead of doing a single integral over $z_{1}, \ldots, z_{N}$. To a limited extent the procedure resembles a random phase approximation, in that each factor $\exp \left(-i x \phi_{i}\right)$ has been averaged over the phase $\phi_{i}$ ignoring any correlation between the different $\phi_{i}$. The accuracy of the approximation will be examined later when we discuss the moments of the distribution.

As a result of the factorization (12) becomes

$$
I_{2}(\Omega)=\frac{1}{2 \pi} \int_{-\infty}^{\infty} d x \exp (i x \Omega)\left[\frac { 1 } { V } \int d z p ( z ) \operatorname { e x p } \left\{-i x \epsilon^{\prime}(z) \epsilon^{\prime \prime}(z)\{\exp \{-\rho \tilde{J}(x, z)\}]^{N} .\right.\right.
$$

The methods of the last section apply, and we may summarize our results in these equations:

$$
\begin{aligned}
I_{2}(\Omega) & =\frac{1}{2 \pi} \int_{-\infty}^{\infty} d x \exp (i x \Omega) \exp \left\{-\rho J_{2}(x)\right\} \\
J_{2}(x) & =\int d z p(z)\left[1-\exp \left\{-i x \epsilon^{\prime}(z) \epsilon^{\prime \prime}(z)-\rho \tilde{J}(x, z)\right\}\right] \\
\tilde{J}(x, z) & =\int d u p(u)\left(1-\exp \left[-i x \frac{1}{2}\left\{\epsilon^{\prime}(z) \epsilon^{\prime \prime}(u)+\epsilon^{\prime}(u) \epsilon^{\prime \prime}(z)\right\}\right]\right) .
\end{aligned}
$$


These results are discussed in the following sections. We confine ourselves to two observations. First the method applies equally well when (2) is generalized to the form

$$
\hbar \omega=\hbar \omega_{0}+\hbar \omega_{2}\left(\epsilon^{\prime} \epsilon^{\prime \prime}+\epsilon^{\prime \prime \prime} \epsilon^{\mathrm{iv}}+\ldots\right)
$$

albeit with an increase in complexity. Secondly the moments can be derived without difficulty. If

$$
M_{n}=\frac{\int_{-\infty}^{\infty} d \Omega \Omega^{n} I_{2}(\Omega)}{\int_{-\infty}^{\infty} d \Omega I_{2}(\Omega)}
$$

then (for details see I)

$$
M_{n}=\lim _{x \rightarrow 0}(-1)^{n} \frac{d^{n}}{d(i x)^{n}}\left[\exp \left\{-\rho J_{2}(x)\right\}\right] .
$$

\section{Alternative second-order theories}

None of the theories described here are new, although none appear to have been discussed critically before. All are approximate.

The first theory makes use of the moments of the distribution of $\Omega$. A small number of the moments are calculated exactly and are used to determine the parameters in an assumed functional form for $I(\Omega)$. For example the functional form might be a Gaussian distribution whose peak is shifted from $\Omega=0$. Two moments are needed here, and these (denoted $\bar{M}_{1}$ and $\bar{M}_{2}$ ) are exact in the sense that they avoid the approximation of $\S 3$. Then

$$
I(\Omega) \sim \exp \left\{-\frac{\frac{1}{2}\left(\Omega-\bar{M}_{1}\right)^{2}}{M_{2}}\right\} .
$$

The moments, $\bar{M}_{1} \equiv\left\langle\epsilon^{\prime} \epsilon^{\prime \prime}\right\rangle$ and $\bar{M}_{2} \equiv\left\langle\epsilon^{\prime} \epsilon^{\prime} \epsilon^{\prime \prime} \epsilon^{\prime \prime}\right\rangle$, can be found from the identities

$$
\begin{aligned}
\left\langle\epsilon^{\prime} \epsilon^{\prime \prime}\right\rangle & =\frac{1}{2}\left\{\left\langle\left(\epsilon^{\prime}+\epsilon^{\prime \prime}\right)^{2}-\left\langle\left(\epsilon^{\prime}\right)^{2}\right\rangle-\left\langle\left(\epsilon^{\prime \prime}\right)^{2}\right\rangle\right\}\right. \\
\left\langle\epsilon^{\prime} \epsilon^{\prime} \epsilon^{\prime \prime} \epsilon^{\prime \prime}\right\rangle & =\frac{1}{12}\left\{\left\langle\left(\epsilon^{\prime}+\epsilon^{\prime \prime}\right)^{4}\right\rangle+\left\langle\left(\epsilon^{\prime}-\epsilon\right)^{4}\right\rangle-2\left\langle\left(\epsilon^{\prime}\right)^{4}\right\rangle-2\left\langle\left(\epsilon^{\prime \prime}\right)^{4}\right\rangle\right\} .
\end{aligned}
$$

Each of the terms on the right-hand sides of these equations can be found by the method of I. The moments are expressed in terms of integrals $S_{n, m}$ :

$$
S_{n, m}=\rho \int d z p(z)\left\{\epsilon^{\prime}(z)\right\}^{n}\left\{\epsilon^{\prime \prime}(z)\right\}^{m}
$$

If $\epsilon^{\prime}$ and $\epsilon^{\prime \prime}$ are identical $S_{n, m}$ is just the $S_{n+m}$ of I. After tedious but straightforward calculation

$$
\begin{aligned}
\bar{M}_{1}= & S_{1,1}+S_{1,0} S_{0,1} \\
= & \rho \int d z p(z) \epsilon^{\prime}(\tau) \epsilon^{\prime \prime}(z)+\rho \int d z p(z) \epsilon^{\prime}(z) \rho \int d u p(u) \epsilon^{\prime \prime}(u) . \\
\bar{M}_{2}= & S_{2,2}+S_{1,0} S_{1,2}+2 S_{0,1} S_{2,1}+2 S_{1,1}{ }^{2}+S_{0,1}{ }^{2} S_{1,0}{ }^{2} \\
& +S_{2,0} S_{0,2}+4 S_{1,1} S_{0,1} S_{1,0}+S_{1,0}{ }^{2} S_{0,2}+S_{0,1}{ }^{2} S_{2,0} .
\end{aligned}
$$

These exact moments give a standard of comparison for second-order theories. For example we may compare $\bar{M}_{1}$ and $\bar{M}_{2}$ with the corresponding moments obtained from the method in $\S 3$. Then $\bar{M}_{1}=\bar{M}_{1}$, so the approximate method gives the exact first moment, but the second moment is not exactly $M_{2}$ :

$$
\begin{aligned}
M_{2}= & S_{2,2}+S_{1,0} S_{1,2}+S_{0,1} S_{2,1}+\frac{3}{2} S_{1,1}{ }^{2} \\
& +\frac{1}{2} S_{0,2} S_{2,0}+\frac{5}{2} S_{1,1} S_{0,1} S_{1,0}+\frac{1}{4} S_{1,0}{ }^{2} S_{0,2}+\frac{1}{4} S_{0,1}{ }^{2} S_{2,0}
\end{aligned}
$$


There are two major disadvantages of the moment method we have been discussing. First we must assume a qualitative form for the line shape. 'The Gaussian (21) is an obvious choice, but it is a gross oversimplification. In particular it does not allow $I(\Omega)$ to be asymmetric about $\Omega=\langle\Omega\rangle$; this is a real restriction, for in cases of practical interest skewed distributions often occur (e.g. the $\Delta M=2$ electron paramagnetic resonance line of $\mathrm{MgO}: \mathrm{Fe}^{2+}$ ). Secondly the $S_{n, m}$ usually diverge at one or more limits of integration. The divergence can be prevented by a cut-off, of course, but the $S_{n, m}$ then depend strongly on the value of the cut-off and this cannot be predicted with accuracy. The method of the last section does not suffer from either of these disadvantages.

The second alternative method is to assume that in

$$
\hbar \omega=\hbar \omega_{0}+\hbar \omega_{2} \epsilon^{\prime} \epsilon^{\prime \prime}
$$

the perturbations $\epsilon^{\prime}$ and $\epsilon^{\prime \prime}$ are statistically independent. This can be generalized to cases where $\epsilon^{\prime}$ and $\epsilon^{\prime \prime}$ are linear combinations of statistically independent perturbations, but this merely introduces complexity. If $\epsilon^{\prime}$ and $\epsilon^{\prime \prime}$ are independent the distribution of $\Omega=\epsilon^{\prime} \epsilon^{\prime \prime}$ can be found from the distributions $I^{\prime}\left(\epsilon^{\prime}\right)$ and $I^{\prime \prime}\left(\epsilon^{\prime \prime}\right)$ of $\epsilon^{\prime}$ and $\epsilon^{\prime \prime}$ respectively:

$$
I(\Omega)=\int_{-\infty}^{\infty} \int_{-\infty}^{\infty} d s d t I^{\prime}(s) I^{\prime \prime}(t) \delta(\Omega-s t) .
$$

This result can be readily applied in practice. In terms of the moments the statistical independence is equivalent to assuming, for example, that $\left\langle\epsilon^{\prime} \epsilon^{\prime \prime}\right\rangle=\left\langle\epsilon^{\prime}\right\rangle\left\langle\epsilon^{\prime \prime}\right\rangle$ which is usually zero. The assumption has been used in another form (McMahon 1964, Feher 1964), when it is assumed that the distribution of $\epsilon^{\prime}+\epsilon^{\prime \prime}$ is given by the appropriate convolution of $I^{\prime}$ and $I^{\prime \prime}$. The objection to this method is that the assumption of statistical independence is not always good. This has already been discussed in $\$ 3.2$ of I, so here we merely give an example. In an isotropic crystal screw dislocations produce no dilatation, so $e_{x x}+e_{y y}+e_{z z}$ is zero; it is clearly incorrect to assume that $e_{x x}, e_{y y}$ and $e_{z z}$ are independent. However, by a judicious choice of $\epsilon^{\prime}$ and $\epsilon^{\prime \prime}$ it may be possible to ensure $\epsilon^{\prime}$ and $\epsilon^{\prime \prime}$ are nearly independent for some symmetry reason, at least sufficiently so for routine analysis.

The third alternative method is, in the present author's view, incorrect. It is mentioned only because it has been used in the literature (equations (24)-(26) of Greenberg 1966). The assumption is to equate $\Omega$ to

$$
\Omega_{1} \equiv \sum_{i=1}^{N} \epsilon^{\prime}\left(z_{i}\right) \epsilon^{\prime \prime}\left(z_{i}\right)
$$

and thus to drop $\sum_{i=1}^{N} \epsilon^{\prime}\left(z_{i}\right) \sum_{j \neq i} \epsilon^{\prime \prime}\left(z_{i}\right)$. The assumption is often poor-in the next section we treat a simple model exactly and find it gives a completely different linewidth and dependence on concentration from (29). Moreover (29) leads to an incorrect first moment as well as incorrect higher moments-only the first term in (25) is given. The only use of (29) is in determining the asymptotic line shape. At very large values of $\Omega$ the important configurations are those in which one defect is close to the centre studied. It may be possible to neglect the contributions to $\Omega$ of all the other defects, so that

$$
\Omega \simeq \epsilon_{0}{ }^{\prime} \epsilon_{0}^{\prime \prime} \simeq \Omega_{1}
$$

where $\epsilon_{0}{ }^{\prime}, \epsilon_{0}{ }^{\prime \prime}$ are the contributions to these perturbations from the nearby defect.

In summary the method of $\S 3$, although approximate, has several advantages. It does not give awkward divergences, nor does it require an arbitrary qualitative choice of line shape. It gives the first moment exactly, and does not rely on the arbitrary assumption that certain perturbations are statistically independent.

\section{Comparison of exact and approximate results}

$I(\Omega)$ can be found only in certain very simple cases. In this section we discuss such a simple case and compare its predictions with the various approximate treatments. Although the model was chosen to make this comparison possible, it has a fairly strong similarity to the case of dislocation broadening. 
The simplifying assumptions are these. The defects only influence one of the centres studied when the defect is within a volume $V$ about the centre. The defect may be in one of four states $i$ in which $\epsilon^{\prime}, \epsilon^{\prime \prime}$ are given below together with the probability $W_{i}$ that the defect is in this state:

$\begin{array}{cccc}\text { state } i & \text { probability } W_{i} & \text { contribution to } \epsilon^{\prime} & \text { contribution to } \epsilon^{\prime \prime} \\ 1 & (1+\gamma) / 4 & s & t \\ 2 & (1-\gamma) / 4 & -s & t \\ 3 & (1-\gamma) / 4 & s & -t \\ 4 & (1+\gamma) / 4 & -s & -t .\end{array}$

The parameter $\gamma$ is a measure of the correlation between $\epsilon^{\prime}$ and $\epsilon^{\prime \prime}$; in fact

where

$$
\gamma \equiv \frac{\left\langle\epsilon^{\prime} \epsilon^{\prime \prime}\right\rangle}{\left\{\left\langle\left(\epsilon^{\prime}\right)^{2}\right\rangle\left\langle\left(\epsilon^{\prime \prime}\right)^{2}\right\rangle\right\}^{1 / 2}}
$$

$$
\langle F\rangle=\sum_{i} W_{i} F(i) \equiv \int d z p(z) F(z)
$$

When $\gamma=0$ the two components are uncorrelated; when $\gamma=1$ the components $\epsilon^{\prime}$ and $\epsilon^{\prime \prime}$ are completely correlated. It should be noted that $\left\langle\epsilon^{\prime}\right\rangle$ and $\left\langle\epsilon^{\prime \prime}\right\rangle$ are zero, independent of $\gamma$.

We further assume that the average number of defects within each volume $V$ is large; if $n$ is the number of defects per unit volume then $n V \gg 1$. Thus the contribution of any one defect to the half-width of the observed line is very small.

There are two broadening mechanisms in our example. One comes from fluctuations in the relative numbers of defects in the different states $i$ which interact with particular centres. The second comes from fluctuations in the total number of defects interacting with any one centre. In the completely correlated case $(\gamma=1)$ only the second mechanism contributes as $\Omega_{i}=s t$ and always has the same sign and magnitude.

Exact solution is possible when there is no correlation in the line shape. In this case $I(\Omega)$ is symmetric about $\Omega=0$ as the states with $\Omega=+s t(i=1,4)$ and those with $\Omega=-s t$ $(i=2,3)$ are equally populated. In this case the important values of $x$ in expressions such as (12) are of order $1 /($ half-width), and as each defect makes only a small contribution to the half-width:

$$
|x s t| \ll 1 \text {. }
$$

The importance of this lies in the fact that the central limit theorem can be used to predict a Gaussian shape for the exact distribution when $n V \gg 1 \gg x s t$ and $\gamma=0$. The exact distribution can then be found from the moments, in particular by (26). In $M_{2}$ we need, for example, $S_{2,2}$ :

$$
S_{2,2}=n V \sum_{i} W_{i}\left(\epsilon_{i} \epsilon_{i}^{\prime \prime}\right)^{2}=n V s^{2} t^{2}
$$

the other terms can be obtained in the same way. The dominant term is $S_{20}, S_{02}=(n V s t)^{2}$, and the full width at half-intensity is

$$
\Delta_{\text {exact }}=2 \sqrt{ } 2(\ln 2)^{1 / 2} n V s t .
$$

This can be written in terms of the width of the distributions of $\epsilon^{\prime}$ and $\epsilon^{\prime \prime}\left(\Delta^{\prime}\right.$ and $\Delta^{\prime \prime}$ respectively):

As expected, this is of order $\Delta^{\prime} \Delta^{\prime \prime}$.

$$
\Delta_{\text {exact }}=\frac{\Delta^{\prime} \Delta^{\prime \prime}}{2 \sqrt{2} \ln 2} .
$$

The approach of $\S 3$ also predicts a Gaussian. By straightforward application of (17)(19) and expansion in powers of xst and $1 / n V$ we find a width

$$
\Delta_{\text {approx }}=2(\ln 2)^{1 / 2} n V s t .
$$


This differs from $\Delta_{\text {exact }}$ by a factor $\sqrt{ } 2$, although it predicts the correct concentration dependence and dependence on $s$ and $t$. This is to be contrasted with the method of Greenberg (1966), which gives a width proportional to $(n V)^{1 / 2}$ and an entirely incorrect concentration dependence. The method based on (28), which assumes that $\epsilon^{\prime}$ and $\epsilon^{\prime \prime}$ are statistically independent, should be exact when $\gamma=0$, but not otherwise. In fact even when $\gamma=0$ the method is less useful than one might hope. The reason is that one normally wishes to use distributions of $\epsilon^{\prime}$ and $\epsilon^{\prime \prime}\left(I^{\prime}\left(\epsilon^{\prime}\right)\right.$ and $I^{\prime \prime}\left(\epsilon^{\prime \prime}\right)$ respectively) which are approximate. In the present model one would use approximations such as $n V \gg 1$ to achieve this. However, the integral in (28) has a sizable contribution from parts of $I^{\prime}(s)$ and $I^{\prime \prime}(t)$ for which the approximations do not hold, and the exact, unwieldy expressions for $I^{\prime}$ and $I^{\prime \prime}$ must be used; in such cases (28) may be intractable. The approximate forms of $I^{\prime}(s)$ and $I^{\prime \prime}(t)$ are not adequate in the model of $(30)$. The resulting line shape is

$$
I(\Omega) \sim \mathrm{K}_{0}\left(\left|\frac{\Omega}{n V s t}-\right|\right)
$$

where $\mathrm{K}_{0}(z)$ is a modified Bessel function. The inadequacy of this result is strikingly shown by the divergence of $\mathrm{K}_{0}(z)$ at small $z$. The divergence is logarithmic, so it does not affect the moments too strongly, but it is a spurious result-there is no real divergence of $I(\Omega)$ at small $\Omega$.

So far we have only considered $\gamma=0$, when $\epsilon^{\prime}$ and $\epsilon^{\prime \prime}$ are uncorrelated. Both the moment method and the method of $\$ 3$ predicted the same dependence of the width on concentration $n V$ and on the defect strengths $(s, t)$. The method of Greenberg gave the wrong concentration dependence, and the approach based on (28) (assuming $\epsilon^{\prime}$ and $\epsilon^{\prime \prime}$ uncorrelated) gave a spurious divergence when normally acceptable approximations for $I_{1}^{\prime}\left(\epsilon^{\prime}\right)$ and $I_{1}^{\prime \prime}\left(\epsilon^{\prime \prime}\right)$ are used. In going to finite $\gamma$ (finite correlation) we still consider only two of these approaches-the moment method and the approach of $\S 3$. The other approaches should be even less successful than they are in the limit $\gamma=0$.

As $\gamma$ increases the first moment of $I(\Omega)$ becomes finite, as defects in states $i=1,4$ become more important. The moment method predicts that $I(\Omega)$ is a Gaussian of width

centred on

$$
\Delta_{\text {Gauss }}=2 \sqrt{ } 2(\ln 2) n V s t\left(1+\gamma^{2}\right)^{1 / 2}
$$

$$
\Omega_{\text {Gauss }}=n V s t \text {. }
$$

As the correlation increases the width increases and the peak of the distribution shifts.

The method of $\S 3$ makes very similar predictions. Near the peak of the distribution the expansions in xst and $1 / n V$ should still hold, and the line shape should be nearly Gaussian, with width

peaked at

$$
\Delta_{\text {approx }}=2(\ln 2)^{1 / 2} n V s t\left(1+\gamma^{2}\right)^{1 / 2}
$$

$$
\Omega_{\text {approx }}=n V s t .
$$

Again the two methods agree in their dependence on the degree of correlation between $\epsilon^{\prime}$ and $\epsilon^{\prime \prime}$.

The consistency of any result can be checked in the limit of complete correlation $\left(\epsilon^{\prime}=\epsilon^{\prime \prime}\right)$ by the analogue of the Kramers-Kronig relation. As $\Omega=\left(\epsilon^{\prime}\right)^{2}$ is never negative,

$$
\pi \mathscr{I}\{F(x)\}=P\left[\int_{-\infty}^{\infty} d s \frac{\mathscr{R}\{F(x)\}}{s+x}\right]
$$

relates the real and imaginary parts of $F(x)$, the Fourier transform of the exact line shape:

$$
I(\Omega)=\frac{1}{2 \pi} \int_{-\infty}^{\infty} d x \exp (i \Omega x) F(x) .
$$


The artificial case discussed in this section is related to broadening by dislocations. This can be seen most easily from the distribution of $\epsilon^{\prime}$ or of $\epsilon^{\prime \prime}$ alone

$$
I_{1}^{\prime}\left(\epsilon^{\prime}\right)=\frac{1}{2 \pi} \int_{-\infty}^{\infty} d x \exp \left(i x \epsilon^{\prime}\right) \exp \left(-\frac{1}{2} n V s^{2} x^{2}\right)
$$

compared with the result for dislocations, omitting a relatively small logarithmic term

$$
I_{\mathrm{d} \mathrm{s} \mathrm{l}}\left(\epsilon^{\prime}\right)=\frac{1}{2 \pi} \int_{-\infty}^{\infty} d x \exp \left(i x \epsilon^{\prime}\right) \exp \left(-L b^{2} A x^{2}\right) .
$$

The dislocation density $L$ replaces the mean number of defects, $n V$, interacting with a centre. The strength of the defect, $s$, is replaced by the Burgers vector $b$ multiplied by a factor $(2 A)^{1 / 2}$ which depends on the component $\epsilon^{\prime}$ considered. All the qualitative features carry over. Thus as $\epsilon^{\prime}$ and $\epsilon^{\prime \prime}$ become more strongly correlated the width changes and the line shape skews. The skewed shape is, of course, exactly that seen in paramagnetic resonance (McMahon 1964) and acoustic resonance (e.g. Lewis and Stoneham 1967).

\section{Second-order effects from dislocations}

This section treats a very practical problem-what is $I(\Omega)$ for strain broadening by dislocations? It will be simplified by assuming this dislocation distribution is isotropic and homogeneous, as described in I. This is only an approximation, for in real crystals subgrain boundaries and other complex structures are often found. We shall also be less ambitious than in $\S 5$, and shall be content with a qualitative description of the line shape and its dependence on parameters such as the dislocation density. Our justification lies in the complexity of the problem; also we recall that $\$ \S 4$ and 5 suggest the theory of $\S 3$ is not very precise for absolute values of widths, but that it is reasonable qualitatively. Moreover the system discussed is too complicated to treat analytically throughout, and a list of computed values gives little insight into the problem.

The first-order problem was solved in I. The distribution of strains $\epsilon$ proves to be of the general form

$$
I(\epsilon)=\frac{1}{2 \pi} \int_{-\infty}^{\infty} d x \exp (i x \epsilon) \exp \left\{L b^{2} x^{2}(A-B \ln |x|)\right\}
$$

in which $L$ is the dislocation density, $b$ the Burgers vector magnitude and $A$ and $B$ are complicated angular integrals. The exponent $L b^{2} x^{2}(A-B \ln |x|)$ is closely related to the $\rho \tilde{J}(x, z)$-in fact they are identical if we write

$$
\epsilon(u)=\frac{1}{2} \epsilon^{\prime}(z) \epsilon^{\prime \prime}(u)+\frac{1}{2} \epsilon^{\prime \prime}(z) \epsilon^{\prime}(u)
$$

treating $\epsilon^{\prime}(u)$ and $\epsilon^{\prime \prime}(z)$ as coefficients. The variables $z$ appear in the coefficients $A$ and $B$. It is the logarithmic term in (40) which causes much of the analytic difficulty in the present problem. Such terms can be dropped, but this must be done with caution. In all cases where this approximation has been made here the error has been estimated by direct numerical integration, using reasonable values of the various parameters.

After considerable manipulation and dropping some small terms we find

$$
\rho J_{2}(x)=\frac{L b^{2}}{8 \pi} \int d \omega \int_{Y}^{\infty} d y \frac{1}{y^{2}}[1-\exp \{-(\Lambda+i \Delta) y\}] .
$$

The angular variables, giving the orientations of dislocation Burgers vectors and axes, are collectively described by $\omega . Y$ is $b^{2} / R^{2}, R$ being the crystal radius and $b$ the Burgers vector, and $y$ is $b^{2} / r^{2} . \Lambda$ and $\Delta$ are functions:

where

$$
\begin{aligned}
& \Lambda=L b^{2} x^{2} F\left(x, \psi^{\prime}, \psi^{\prime \prime}\right) \\
& \Delta=x \psi^{\prime} \psi^{\prime \prime} / \pi^{2}
\end{aligned}
$$

$$
F=\frac{7 \cdot 8108-\ln \left\{(b x / R)^{2}\left(\left|\psi^{\prime}\right|^{2}+\left|\psi^{\prime \prime}\right| 2\right)\right\}}{64 \pi^{2}}
$$


and

$$
\psi^{\prime}(\omega)=\frac{\epsilon^{\prime}(\omega, r)}{b / 2 \pi r}
$$

$F\left(x, \psi^{\prime}, \psi^{\prime \prime}\right)$ does not vary rapidly with the variables on which it depends.

The upper limit of $\infty$ in the innermost integral of (41) means we are ignoring lattice structure and integrating from $r=0$ outwards. As $Y \equiv b^{2} / R^{2}$ is small (typically $10^{-14}$ ) we may also assume $R$ infinite, except in those terms which diverge logarithmically. The result is

$$
\begin{aligned}
\rho J_{2}(x)= & \frac{L b^{2}}{8 \pi} \int d \omega\left[\left\{\Delta \tan ^{-1}\left(\frac{\Delta}{\Lambda}\right)-\Lambda \ln \left(\frac{\gamma Y\left(\Lambda^{2}+\Delta^{2}\right)^{1 / 2}}{\mathrm{e}}\right)\right\}\right. \\
& \left.+i\left\{\Lambda \tan ^{-1}\left(\frac{\Delta}{\Lambda}\right)+\Delta \ln \left(\frac{\gamma\left(\Lambda^{2}+\Delta^{2}\right)^{1 / 2}}{\mathrm{e}}\right)\right\}\right]
\end{aligned}
$$

where $\gamma$ is Euler's constant $(\ln \gamma=0.57721 \ldots)$ and $e=2.718 \ldots$ This is still too complicated to evaluate exactly. Instead we take two extreme limits. Both limits can be achieved at reasonable dislocation densities. In the high-density limit $\Lambda \gg \Delta$ the integrand becomes

$$
-\Lambda\left\{\ln \left(\frac{\gamma Y}{\mathrm{e}}\right)+\ln \Lambda\right\}-i \Delta\left\{1+\ln \left(\frac{\gamma Y}{\mathrm{e}}\right)+\ln \Lambda\right\} \text {. }
$$

As In $\Lambda$ varies relatively slowly with $x$ we can treat it as a constant. The first term is proportional to $L b^{2} x^{2}$, so the real part of $\rho J_{2}$ varies as $L^{2} x^{2} b^{2}$. Thus we expect a Gaussian line with width proportional to $L b^{2}$. The second term is proportional to $x$, so the peak of the Gaussian should be shifted from $\epsilon^{\prime} \epsilon^{\prime \prime}=0$ by an amount proportional to $L b^{2}$. In the opposite limit $\Delta \gg \Lambda$, which holds at lower densities and also in the extreme wings of $I(\Omega)$, the integrand becomes

$$
\frac{1}{2} \pi \mid+i \Delta \ln \left(\frac{\gamma Y \Delta}{\mathrm{e}}\right) \text {. }
$$

In this case $\rho J_{2}$ has a real part proportional to $L b^{2}|x|$ and an imaginary part proportional to $L b^{2} x$. The line is Lorentzian, with width and shift linear in $L b^{2}$.

In both these extreme cases the width and the shift are proportional to the dislocation density $L$ and the square of the Burgers vector. The shape varies from Lorentzian to Gaussian as $L$ increases; at intermediate values of $\Lambda / \Delta$ the line will not be so simple and may be skewed. In fact the skewness will show whenever $\mathscr{I}\left\{J_{2}(z) / x\right\}$ varies with $x$-only when this is independent of $x$ is there a rigid shift. The weakness of the moment method can also be demonstrated, as both the first and second moments (related to the shift and width respectively) diverge unless a cut-off is included at both large and small $r$.

\section{Conclusion}

This paper described methods of treating second-order inhomogeneous broadening of resonance lines, and a new approximate extension of the statistical method was developed to deal with these cases. The new method offers advantages over the previous methods: it does not rely on qualitative estimates of the line shape, it is valid for any second-order term (and not just the product of two uncorrelated first-order terms) and it predicts the correct qualitative dependence on defect strengths and concentrations. The various approaches were compared for a simple model and the case of strain broadening by dislocations.

\section{References}

FEHER, E. R., 1964, Phys. Rev., 136, A145-57.

Greenberg, B., 1966, Phys. St. Solidi, 17, 673-81.

Kawamura, H., Orsuka, E., and Ishiwatari, K., 1956, J. Phys. Soc. Japan, 11, 1064-77.

Iewis, M. F., and Stoneham, A. M., 1967, Phys. Rev., 164, 271-84.

McMahon, D., 1964, Phys. Rev., 134, A128-39.

Markham, J. J., 1966, F Centres in Alkali Halides (New York: Academic Press).

Stoneham, A. M., 1966, Proc. Phys. Soc., 89, 909-21. 